\title{
Representaciones sociales de la educaciòn inicial
}

\section{Social representations of early education}

\author{
Mónica Janeth Ramírez-Botero \\ Beatriz Elena Arias-Vanegas ${ }^{2}$ \\ Renato Zambrano-Cruz ${ }^{3}$ \\ Grupo Interdisciplinario de Estudios \\ Pedagógicos GIDEP
}

\section{RESUMEN}

La educación inicial de los hijos es una de las prioridades que deben resolver los padres, bien sea porque ellos se ocupen de su educación

\begin{tabular}{l}
\hline 1 Magíster, Maestría en educación \\
Secretaría de Educación de Medellín \\
Grupo de investigación: Grupo Interdisciplinario de Estudios \\
Pedagógicos GIDEP Medellín-Colombia \\
$\frac{\text { Monica.ramirezbotero@medellin.edu.co; }}{\text { monicajanethramirez@gmail.com }}$ \\
ORCID: $\frac{\text { https://orcid.org/0000-0002-3737-5733 }}{\text { académico: } \underline{\text { https://scholar.google.es/ }}}$ \\
citations?user=-B2eDrEAAAAJ\&l=es
\end{tabular}

2 Doctora, Doctorado en Ciencias Sociales: Niñez y Juventud Universidad de San Buenaventura - Medellín Grupo de investigación: Grupo Interdisciplinario de Estudios Pedagógicos GIDEP Medellín-Colombia betyarv@gmail.com ORCID: https://orcid.org/0000-0003-0432-8532 Google académico: https://scholar.google.com/citations?us er=1SyqqQkAAAAJ\&hl=es

3 Magíster, Maestría en lingüística Universidad Cooperativa de Colombia, seccional Medellín Neurociencia y Cognición Medellín - Colombia renato.zambrano@ucc.edu.co ORCID: https://orcid.org/0000-0003-2155-0039 Google académico: https://scholar.google.es/ citations? user=vc-GrYwAAAAJ\&hl=es en el entorno familiar o porque la deleguen. Este artículo surgió del doctorado en ciencias de la educación de la Universidad de San Buenaventura Medellín y buscó comprender las representaciones sociales (RS) acerca de la educación inicial que tienen los padres de niños de primera infancia de la ciudad de Medellín. Este estudio tuvo un enfoque fenomenológico y se desarrolló en el marco de las RS utilizando el grupo focal y la entrevista semiestructurada. Se recolectó la información con 20 personas, resultando 617 códigos agrupados en 15 categorías que configuraron las RS de la educación inicial y que determinaron como núcleo central los efectos de la institucionalización. Se concluyó que los padres tienen percepciones positivas de las instituciones, las profesoras y los programas de educación inicial de Medellín. Las RS de la educación inicial de los padres están 
influenciadas por sus exigencias laborales, confiando la educación a las profesoras.

\section{PALABRAS CLAVE}

Padres (progenitores); Primera infancia; Institucionalización; Educación de la primera infancia; Psicología social.

\section{SUMMARY}

The early education is a priority that parents must solve, either because they take care of their education in the family environment or because they delegate it. This paper arose from the doctorate in educational sciences of the University of San Buenaventura Medellín and sought to understand the social representations (SR) about the early education that parents of early childhood have in the city of Medellín. This study had a phenomenological approach and was developed within the SR framework using the focus group and the semi-structured interview. The information was collected with 20 people, the outcomes shows 617 codes grouped in 15 categories that configured the SR of early education and that determined the effects of institutionalization as the central core. It was concluded that parents have positive perceptions of the institutions, the teachers and early education programs in Medellín. The SR of parents' about early education are influenced by their job demands, entrusting the education to the teachers.

\section{KEYWORDS}

Parents; Early childhood; Institutionalization; Early childhood education; Social psychology.

\section{INTRODUCCIÓN}

Escolarizar a los niños desde antes de los seis años de vida se ha tornado en una práctica social cada vez más fuerte; lo que inició con la idea de crear jardines infantiles para compensar la ausencia de la mujer en el hogar por su vinculación al campo laboral, se ha convertido en la estrategia para favorecer el desarrollo de los niños, prevenir situaciones de abandono que generan a la sociedad costos altos de intervención psicosocial que terminan siendo mayores a la inversión que pueda hacerse en la primera infancia.

En los últimos 20 años, la región latinoamericana ha aumentado en gran volumen la cobertura de los servicios de cuidado a la primera infancia, que son básicamente jardines de infantes para niños de 0 a 3 años (Berlinsky y Schady, 2015). El incremento en la cobertura de atención a la primera infancia que se observa en países como Chile, Brasil, Uruguay, Ecuador, Colombia ha sido en $100 \%$, lo que quiere decir que en los últimos años se ha duplicado de tal manera que alrededor de un cuarto de los niños entre $0 \mathrm{y}$ 3 años están en este momento en jardines de infantes, y la fracción es mayor entre los niños de 3 a 5 años en comparación con los que tienen menos de un año (Banco Interamericano de Desarrollo BID, 2015).

Sin embargo es importante señalar que en el impacto de los servicios de atención y cuidado a la primera infancia sobre el desarrollo, influye mucho la calidad y el tipo de interacciones que se generan entre los niños y las personas que se encargan de cuidarlos. Los informes de evaluación de política pública para América Latina muestran que en general la calidad del servicio es muy baja, entendiendo esta valoración en dimensiones críticas, en particular en la dimensión que mide la interacción entre las cuidadoras y los niños en los centros de atención a la primera infancia. Cuando se evalúa la calidad de la atención a la primera infancia uno de sus componentes fundamentales son los procesos y estos hacen referencia a dos dimensiones, la primera son las interacciones con los cuidadores y la otra dimensión es el desarrollo de los niños. Estas dimensiones son las más críticas y las que verdaderamente 
tienen un efecto más fuerte en lo que se refiere al desarrollo de los niños que se benefician de estos servicios.

La información presentada por el BID en el 2015 define la calidad de la atención a los niños en una escala del 1 al 7; la investigación permite evidenciar que la mayoría de los centros en los cuales están estos niños en los países de Latinoamérica se ubican en un rango de 1 o 2, siendo éstos servicios de muy baja calidad, y muy pocos se encuentran en servicios de calidad intermedia, 3, 4 y 5 . En la mayoría de los países prácticamente ningún niño de los que evalúan las encuestas se encuentra en servicios de calidad alta, 6 o 7 .

El informe reporta investigaciones de calidad aplicadas por los mismos estados con la asesoría de organizaciones internacionales como Save the Children, en el caso de Chile, Brasil, Perú, Bolivia, Ecuador se evidencia que estos servicios de cuidado a la primera infancia son en general extremadamente bajos, es decir que se encuentran en una escala entre 1-2. La medición que hizo el Ministerio de Educación Nacional en Colombia entre el 2015 y 2017 evidencia que la calidad de la atención a primera infancia se encuentra en la mayoría de las dimensiones evaluadas entre 3-4.

Lo que ha sucedido en la región es que la ampliación de la cobertura de los servicios de atención integral a la primera infancia, en buena medida, ha respondido a una presión para facilitar el ingreso de las mujeres en el mercado laboral. Esto, obviamente, es un objetivo deseable, que las mujeres si quieren trabajar tengan las facilidades para hacerlo, pero entonces, es necesario establecer una relación lógica entre cobertura y calidad de la atención a la primera infancia. De otra manera, lo que sería una ganancia para las mujeres podría no beneficiar a los niños de primera infancia y podría traer consecuencias mayores en términos de desarrollo humano para las generaciones siguientes.

En Colombia, la aparición de la educación preescolar generó el imaginario en las familias de que los niños debían ingresar lo más rápido posible a las instituciones que prestaban dicho servicio (Cerda, 1996), relegando el rol de la educación y la crianza a dichas entidades y dejando atrás la tradición que había existido por muchos años donde la atención, el cuidado, la socialización y la educación del niño pequeño dependían exclusivamente de la familia.

En consecuencia, la institucionalización de los niños en su primera infancia se ha convertido en la respuesta a múltiples problemas que afectan a la infancia en general, tales como la alimentación y el cuidado mientras los padres de familia trabajan. En primera infancia tiene mayor efecto puesto que es el grupo poblacional más afectado por el maltrato, con cifras nacionales en 2017 se registraron 4.228 ingresos al programa de restitución de derechos del Instituto Colombiano de Bienestar Familiar (ICBF), cifra superior en un 30\% al de niñez (6-11 años de edad $)^{4}$, respecto a la alimentación, se encuentra que este grupo poblacional tiene una prevalencia de retraso en talla del $13,2 \%$ y de $0,9 \%$ en desnutrición aguda, esto difiere por regiones, en las que Antioquia tiene un registro más positivo frente a otras áreas del país (MINSALUD, 2016). Estas problemáticas mencionadas, harían que se represente a las instituciones como garantes nutricionales y de seguridad física y psicológica, sin embargo, ello no está claro en los diversos estudios. La institucionalización de los niños es una decisión que deben tomar los padres de familia sin importar las condiciones económicas a las que pertenezcan, esta decisión estaría influenciada por las convicciones, creencias, percepciones y representaciones que tienen sobre una institución que les ofrezca cuidado y educación.

\footnotetext{
$4 \quad$ https://www.icbf.gov.co/sites/default/files/infografia_maltrato_observatorio.pdf
} 
Si bien es abundante la investigación de RS en contextos escolares con más de 2 millones de publicaciones en google académico a junio de 2020, se han encontrado pocos estudios específicos sobre RS de los padres acerca de la educación inicial de los niños.

En Finlandia, Räty y Snellman (1998) realizaron dos estudios con padres y profesores sobre las representaciones sociales de la educabilidad. Fueron 438 profesores de escuela (tanto primaria como secundaria) y 563 padres de niños de 9 o 10 años. Se indagaron con un cuestionario tipo Likert ad hoc de 60 oraciones acerca de temas relacionados al debate educativo finés y se realizaron análisis factoriales y comparaciones estadísticas de las respuestas según el estado socioeconómico.

Encontraron que hay dos ideas de la educación que subyacen a las representaciones sociales de los padres, una relacionada con la visión selectiva de la educación relacionada con la superdotación natural y otra idea relacionada con la visión comprensiva de la educación, relacionada con la igualdad social. Ambas ideas muestran un vínculo estrecho con la concepción de la inteligencia (natural o social). También hallaron que las respuestas se agrupan en cuatro factores confiables: a. ideología de la superdotación, b. aspectos de inequidad y competición, c. efectividad del mercado y d. funcionamiento de la escuela comprensiva. En la comparación respecto al estado socioeconómico se encontraron diferencias significativas en todas las dimensiones. Respecto a los padres, había mayor preocupación por los aspectos de inequidad y competición en la clase baja, así como una valoración más favorable del sistema escolar comprensivo.

En una réplica mejorada del estudio anterior, Räty, Komulainen y Hirva (2012) realizaron un amplio estudio nacional pero solamente en padres finlandeses acerca de las RS de la educabilidad. Fueron 642 padres de niños de 9 o 10 años y se indagaron con un cuestionario tipo Likert ad hoc de 65 oraciones acerca de temas relacionados al debate educativo finés que tuvo inspiración en el estudio de 1998. Se realizaron análisis factoriales y comparaciones estadísticas de las respuestas según género y nivel académico. Encontraron que las respuestas de los padres se agrupan en siete factores: a. ideología de la superdotación, b. problemas de la educación inmigrante, c. inequidad y competición, d. educación en emprendimiento, e. apoyo para la equidad social y multiplicidad de diferencias humanas, f. principios de mercado y g. que el sistema de escuela comprensivo funciona bien. Los participantes mostraron gran apoyo por la equidad social y las diferencias humanas, así como una evaluación positiva de que el sistema educativo comprensivo funciona bien. En contraste, se oponen a las ideas de enseñanza de los principios del mercado así como explican que la inmigración causa problemas en la educación.

Adicionalmente, encontraron diferencias estadísticamente significativas según sexo $y$ nivel académico. Según el sexo, las madres están más preocupadas por la inequidad que los padres, así como también se preocupan por tener mayor apoyo por la igualdad social y consideran que el sistema escolar comprensivo funciona bien; los hombres, en cambio, tienen mayor afinidad por una ideología de la superdotación y los principios del mercado. En relación con el nivel académico, los padres con formación de nivel superior se inclinaban más por la ideología de la superdotación, mientras que los de nivel más bajo consideraban que la inmigración y la educación multicultural generaban más problemas, aunque apoyaban la igualdad social. Los autores concluyen discutiendo que el abordaje sobre la educación que tienen los padres está estructurado en dos representaciones de la inteligencia (la social y la natural) y que esto direcciona las elecciones de escuela, así como el apoyo a diferentes 
políticas educativas. Abordan la importancia de futuras investigaciones sobre la inmigración y la educación tanto en su país como en otros sistemas educativos.

En Francia, Fontaine y Hamon (2010) realizaron un estudio de representaciones sociales de la escuela en padres y profesores de la Isla Réunion, un territorio francés de ultramar localizado en África. Para realizar el estudio se basaron en la teoría del núcleo central, por lo que utilizaron un cuestionario construido desde el trabajo de Abric y Vergès. Evaluaron a 511 sujetos y analizaron los datos de acuerdo a si eran padres o padres-profesores de primaria y a si eran originales de Réunion o de Francia metropolitana. En el grupo de padres encontraron que el núcleo central estaba sobre la base de los aprendizajes y la educación, la primera periferia tenía que ver con la lectura y la escritura, así como conocimientos, enseñanza, trabajo y éxito, mientras que la segunda periferia está asociada con el respeto, la disciplina y la diplomacia. Los autores discuten que estos hallazgos podrían orientar el trabajo con la comunidad educativa con el fin de aumentar la calidad de la educación en su contexto.

Limón-Aguirre (2011) realizó un estudio sobre las RS de la educación escolar entre los chuj mexicanos, aportando a los interesados en la educación básica con relación a interculturalidad o educación indígena. Se realizaron entrevistas semiestructuradas a un porcentaje entre $40 \%$ y $80 \%$ de los hogares de cada comunidad orientadas a los temas de educación, escuela y los conocimientos culturales; así mismo se dialogó acerca de estos temas con los profesores y los estudiantes de las escuelas primarias. Inicialmente se analizó la información que aportó la comunidad y el sector educativo encontrando devaluación y desprecio por todo lo relacionado con los chuj al lado de la mexicanización obligada, una nula relación entre la escuela y la comunidad en términos de proyectos, la exaltación de la lectoescritura en español y la intención, sin más, de que los alumnos se incorporen al nivel inmediato superior al que se encuentran. Seguidamente se analizó el campo representacional de los chuj mexicanos en relación con la educación escolar, demarcado básicamente a aprender a hablar, leer y escribir en español.

Por último, Rogero-García y Candelas (2015) abordaron las RS de los padres y madres sobre la educación pública y privada en España con el objetivo de conocer si existen RS diferenciadas para los centros públicos y para los centros privados, así como para identificar si tales representaciones sociales varían según la situación socioeconómica de las familias. Se utilizó una muestra de 837 personas con hijos matriculados en los niveles educativos obligatorios hasta el ingreso a la universidad, aplicando una encuesta que demostró que estos padres conciben de manera diferente los centros públicos y privados de acuerdo con su nivel socioeconómico, ideología, religión y lugar de residencia. La mayoría asocia la idea de justicia en el reconocimiento del mérito a la educación pública, mientras que la empleabilidad, la exigencia y la integralidad de la educación la asocian más a la educación privada.

De los pocos estudios específicos encontrados se concluye que existen dos grandes ideas relacionadas con las RS de la educación, una ligada a aspectos de lectura y escritura y conocimientos específicos (Fontaine y Hamon, 2010; Limón-Aguirre, 2011), mientras que las otras están ligadas a aspectos de la equidad, la calidad de la educación y las diferencias individuales (Räty y Snellman, 1998; Räty, Komoulainen y Hirva, 2012; Rogero-García y Candelas, 2015). Los hallazgos muestran que el núcleo central de las representaciones está marcado por un fuerte énfasis en la equidad, el reconocimiento de diferencias individuales y la importancia de los aprendizajes, mientras que 
en la periferia se establece la importancia de la lectura y escritura, así como de conocimientos específicos. Surgió una segunda periferia, en la que aparecieron ideas relacionadas con aspectos de socialización, de disciplina, de convivencia y similares (ver figura 1).

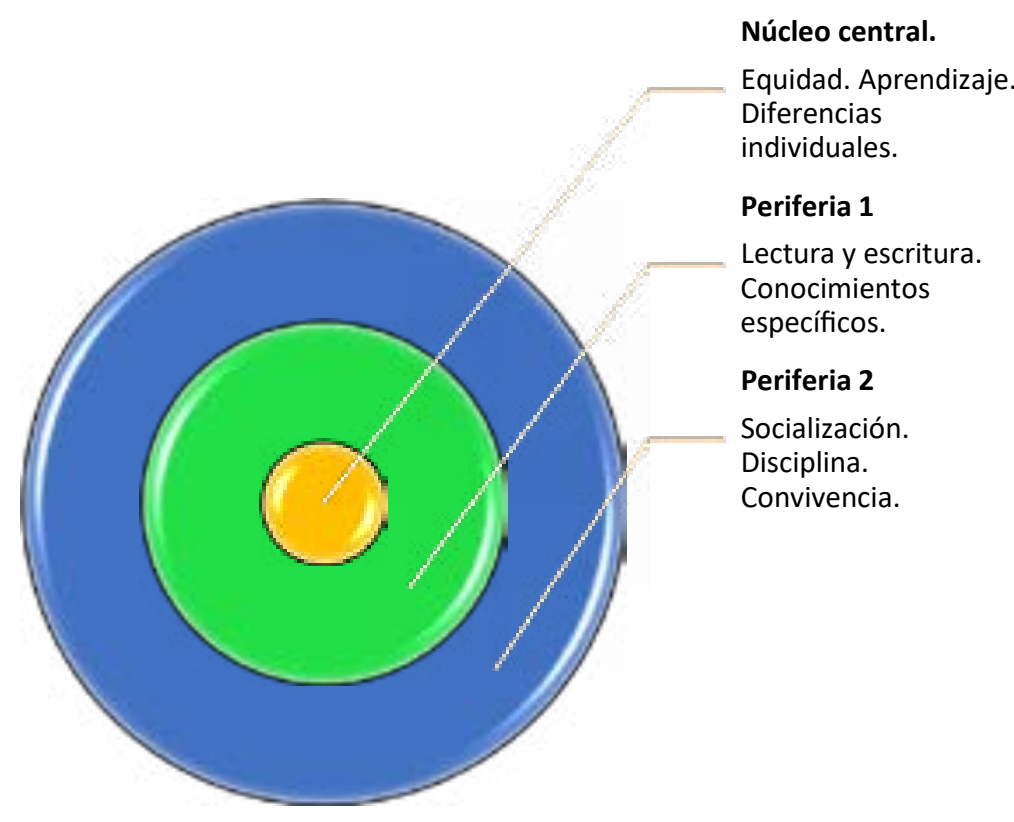

Figura 1. Síntesis de antecedentes investigativos. Fuente: Autores.

A pesar de ser estudios específicos de las representaciones sociales de la educación realizados con padres, estos no son de educación inicial o preescolar sino de primaria o secundaria, por lo que no abordan aspectos de la educación inicial, sino que tienden a ser genéricos y no específicos como sí lo indagó esta investigación.

Siguiendo la línea conceptual de Abric (2001), una RS se constituye de un conjunto organizado y estructurado de informaciones, de creencias, de opiniones y de actitudes a propósito de un objeto dado, es decir, la representación se organiza de manera interna para ir dándole significado al fenómeno social que se esté estudiando. Las representaciones son apropiadas por los individuos y los grupos, la representación funciona como un sistema de interpretación de la realidad que rige las relaciones de los individuos con su entorno físico y social, y que determinará sus comportamientos o sus prácticas (Abric, 2001).

La representación se organiza de forma jerarquizada alrededor de un núcleo central, donde cada uno de los elementos va dando significación a la representación, otorgándole mayor estabilidad a dicho núcleo y donde los elementos que le siguen en orden de importancia (periféricos) permiten que se observen variaciones en el fenómeno social que se esté estudiando (Abric, 2001). El sistema o núcleo central es muy importante en la estabilidad de la representación y éste garantiza su conservación en el tiempo; mientras que el sistema periférico se va formando alrededor y permite algunas RS más individualizadas que dan cuenta de procesos de transformación que puedan ocurrir. De esta manera, estudiar las RS permite comprender la interacción entre la individualidad y las condiciones sociales en 
las que interactúan las personas. Del mismo modo se percibe la manera como los individuos se adaptan a las realidades cotidianas y a las características del entorno.

Abric (2001) plantea que las RS son alcanzadas por consenso y a la vez marcadas por fuertes diferencias interindividuales, es decir, que el estudio de las RS debe, por tanto, tener en cuenta las diferencias interindividuales $e$ ir determinando si esas diferencias son esenciales para ir organizándolas alrededor de un núcleo central. Es en este sentido que el estudio de las RS adquiere mucha importancia porque ofrece un marco de análisis y de interpretación que permite entender la interacción entre el funcionamiento individual y las condiciones sociales en que los actores sociales evolucionan. Es por ello por lo que se pueden entender los procesos que intervienen en la adaptación psicosocial de los individuos a las realidades cotidianas y a las características de su entorno social e ideológico, tanto de padres de familia que tienen la escolaridad de la educación inicial como opción indispensable en la vida de sus hijos, como los padres que promueven la educación en casa.

Conocer las RS de los padres con relación a la educación inicial fue necesario porque permitió descubrir las razones que ellos tenían para institucionalizar a sus hijos en los primeros años de vida, tanto los que estaban de acuerdo con la educación inicial que se ofrece en las instituciones que atienden a los niños de primera infancia en la ciudad de Medellín, como los que decidieron educar en casa.

Es así como este trabajo buscó comprender el significado de las representaciones sociales que tienen los padres y las madres de familia de los niños de primera infancia de la ciudad de Medellín acerca de la educación inicial.

\section{MÉTODO}

El interaccionismo simbólico da origen a la pregunta por el conjunto común de símbolos que surgen para darle sentido a las interacciones de las personas y la importancia que estas tienen en la construcción del conocimiento; y para esta investigación sus aportes más importantes tienen que ver con el significado y la interpretación como procesos humanos esenciales, donde las personas crean significados compartidos a través de su interacción $\mathrm{y}$, estos significados devienen de su realidad, en este caso, de las vivencias en educación inicial que tienen los padres de familia.

Durkheim (2001), plantea las representaciones colectivas, haciendo una diferencia entre estas y las representaciones individuales, explicando que lo colectivo no se reduce a lo individual, lo colectivo se visibiliza en las creencias y demás productos culturales; incorpora el concepto de representaciones colectivas para dar cuenta del pensamiento social, es decir, de las formas de pensamiento compartidas por varios individuos, trascendiendo las representaciones que tenga cada persona. Sin embargo, es Moscovici, (1979) quien introduce el concepto de RS, refiriéndose a ellas como un conjunto de conceptos, enunciados y explicaciones originados en la vida diaria, en el curso de las comunicaciones interindividuales; de acuerdo con Moscovici las RS no son sólo productos mentales, sino que el autor habla de construcciones simbólicas que se crean y recrean en el curso de las interacciones sociales.

Sobre los aportes de Moscovici, Abric (2001) afirma que las RS son apropiadas por los individuos y los grupos, diciendo que la representación funciona como un sistema de interpretación de la realidad que rige las relaciones de los individuos con su entorno 
físico y social, ya que determinará sus comportamientos o sus prácticas (Abric, 2001, p. 13).

Para estudiar las RS en esta investigación se integraron las estrategias de (Abric, 2001, p. 54; Rateau, Moliner, Guimelli y Abric, 2011), donde se proponen tres etapas sucesivas: 1. Identificación del contenido de la representación; 2. Relaciones entre los elementos, su importancia y jerarquía y 3 . Determinación y control del núcleo central. A partir del enfoque cuantitativo planteado por estos autores, se resignificó el enfoque metodológico por un trabajo cualitativo más profundo, cuyas estrategias se describen a continuación con el fin de comprender las $\mathrm{RS}$ de los padres y madres con relación a la educación inicial, de esta manera el estudio exigió profundizar en el diálogo con los padres acerca del sentido y la construcción significativa de sus propias representaciones.

\section{PARTICIPANTES}

Se seleccionaron 20 padres de familia (uno de cada grupo familiar) de las diferentes zonas urbanas en las que se divide la ciudad de Medellín, de manera no probabilística. Considerando como criterios de inclusión de los participantes en esta investigación que fueran padres o madres de niños de primera infancia que iniciaban su proceso educativo y que pertenecieran a diferentes estratos sociales.

\section{ESTRATEGIAS DE RECOLECCIÓN DE INFORMACIÓN}

Inicialmente se realizó el grupo focal con padres y madres de niños de primera infancia de diferentes zonas de la ciudad de Medellín, para indagar por los núcleos de significación de la educación inicial. Con esta técnica del grupo focal se reunieron a los participantes procurando que el grupo de padres y madres de niños de primera infancia discutieran y aportaran, desde la experiencia personal, acerca de la educación inicial de los niños como objeto de investigación. Teniendo en cuenta que la información que se obtuvo proviene de la interacción de los participantes y en esencia es el interés del investigador lo que provee el foco (Morgan, 1997; Morgan y Krueger, 1998), se realizaron unas preguntas orientadoras que configuraron el núcleo central de las RS.

Las categorías que se indagaron en el grupo focal se basaron en la propuesta de Abric (2001) acerca de la organización de las RS. Se diseñaron 2 preguntas por cada uno de los factores que fueron configurando el núcleo central, los cuales fueron: a. naturaleza del objeto representado, b. relación de la persona o grupo con dicho objeto, y c. sistema ideológico que enmarca la relación entre el sujeto y el objeto. Así mismo, se fue definiendo el sistema periférico a través de dichas preguntas, teniendo en cuenta que algunos componentes se iban ubicando más cercanos o lejanos al núcleo (Restrepo-Ochoa, 2013; Abric, 2001). 
Tabla 1. Guía para el desarrollo de grupo focal

\begin{tabular}{|c|c|}
\hline Factores o categorías & Preguntas orientadoras \\
\hline Naturaleza del objeto representado. & $\begin{array}{l}\text { 1. ¿Cuál creen usted qué es la } \\
\text { intencionalidad de la educación inicial? } \\
\text { 2. ¿En qué consiste la educación que } \\
\text { se les ofrece a los niños de primera } \\
\text { infancia? }\end{array}$ \\
\hline Relación de la persona o grupo con dicho objeto. & $\begin{array}{l}\text { 1. ¿Cómo vivió usted sus primeros años } \\
\text { de educación, es decir, qué es lo que } \\
\text { más recuerda de sus primeros años de } \\
\text { educación? } \\
\text { 2. ¿Cómo ha vivido usted los primeros } \\
\text { años de educación de su hijo? }\end{array}$ \\
\hline $\begin{array}{l}\text { Sistema ideológico que enmarca la relación entre el } \\
\text { sujeto y el objeto. }\end{array}$ & $\begin{array}{l}\text { 1. ¿Qué cree usted que se deben enseñar } \\
\text { a los niños en la educación inicial? } \\
\text { 2. ¿Cuáles estrategias de formación se } \\
\text { deben utilizar en la educación inicial? }\end{array}$ \\
\hline
\end{tabular}

Posteriormente se profundizó en las experiencias de los participantes continuando con la perspectiva fenomenológica, desde la cual un fenómeno puede ser reconstruido, a partir de la estructuración que hacen en su conciencia los sujetos que han tenido la experiencia con él. La información más detallada se recolectó con los mismos participantes del grupo focal y se utilizó como técnica de recolección de información la entrevista semiestructurada con el propósito de obtener mayor conocimiento de las RS de los padres de familia con respecto a la educación inicial de los niños. Esta estrategia fenomenológica se realizó indagando por los contenidos que aparecieron en el grupo focal, esto es, sobre los contenidos del núcleo central y los del sistema periférico.

Tabla 2. Guía de entrevista semiestructurada

\begin{tabular}{|l|l|}
\hline $\begin{array}{l}\text { Factores o categorías } \\
\text { Naturaleza del objeto representado. }\end{array}$ & $\begin{array}{l}\text { Preguntas orientadoras } \\
\text { 1. ¿Qué piensa usted de las instituciones que } \\
\text { ofrecen atención a los niños de primera infancia? } \\
\text { 2. ¿Para qué sirve la educación inicial en los } \\
\text { niños? } \\
\text { Relación de la persona o grupo con dicho ¿Cuáles fueron las razones por las que usted } \\
\text { objeto. } \\
\text { decidió escolarizar a su hijo (ofrecer educación } \\
\text { en casa)? } \\
2 . \text { ¿Qué espera usted de la educación inicial que } \\
\text { se les ofrece a los niños en las instituciones que } \\
\text { atienden a la primera infancia (que ofrece a su } \\
\text { hijo en casa)? } \\
\text { 1. ¿De qué manera influye la educación inicial en } \\
\text { el futuro de los niños? } \\
\text { 2. ¿Cómo debe ser la educación inicial que } \\
\text { reciben los niños en los primeros años de vida? }\end{array}$ \\
\hline
\end{tabular}




\section{CONSIDERACIONES ÉTICAS}

Se consideraron las normas de investigación con humanos para estudios psicológicos, puesto que el dominio de las RS se encuentra en la disciplina psicológica y los autores son psicólogos. Se actuó bajo la ley 1090 de 2006, específicamente los artículos del 22 al 31 , donde se estipula el manejo de la información en evaluaciones psicológicas, así como los artículos 49, 50, 51, 55 y 56, en los que se aborda el manejo de investigaciones. Esta investigación fue de riesgo mínimo para los seres humanos de acuerdo con la resolución 8430 de 1993 del Ministerio de Salud.

\section{PLAN DE ANÁLISIS DE INFORMACIÓN}

Se siguieron los pasos de la teoría fundamentada mediante el software Atlas.ti 8.4. Se realizó un proceso de tres niveles de categorización: 1. Codificación abierta. 2. Codificación axial. 3. Codificación selectiva.

\section{RESULTADOS}

Al analizar los testimonios de los participantes, resultaron 617 códigos que se agruparon de acuerdo con el proceso de codificación descrito en el plan de análisis. Una vez obtenidos los grupos de códigos, se organizaron de acuerdo con la teoría de Abric (2001) de forma jerárquica y teniendo en cuenta la acumulación de testimonios similares expresados por los padres de familia.

Construimos 15 categorías mediante las que configuramos las RS de la educación inicial. En el núcleo central de la RS aparecen los efectos de la institucionalización, mientras que en la primera periferia aparecen la relación con los profesores, los argumentos para institucionalizar, la percepción de las instituciones, argumentos para educar en casa y el ideal de educación. En la segunda periferia aparecen las categorías de acompañamiento de padres, vínculo con el hijo, contenidos de la educación inicial, métodos para la educación inicial, educación inicial, estrategias de regulación emocional, manejo de los niños, percepción del niño y relación con los otros niños.

Las categorías más alejadas del núcleo central tienen que ver con la percepción que los padres de familia tienen de sí mismos y su influencia en la educación de sus hijos; así mismo aparecen muchos elementos relacionados con la educación inicial de los niños que dan cuenta de conocimiento que ellos tienen de todo lo relacionado con los procesos educativos. Dicha apreciación estabiliza a la primera periferia que se refiere específicamente a las situaciones que se viven en los contextos escolares y a las personas que ofrecen el servicio de educación inicial en la ciudad de Medellín, permitiendo argumentar las ventajas de la institucionalización con relación a la educación en casa. La percepción positiva de las instituciones de educación inicial expresada en los testimonios de los padres de familia, consolida los efectos de la institucionalización como núcleo principal de esta investigación (ver figura 2). 


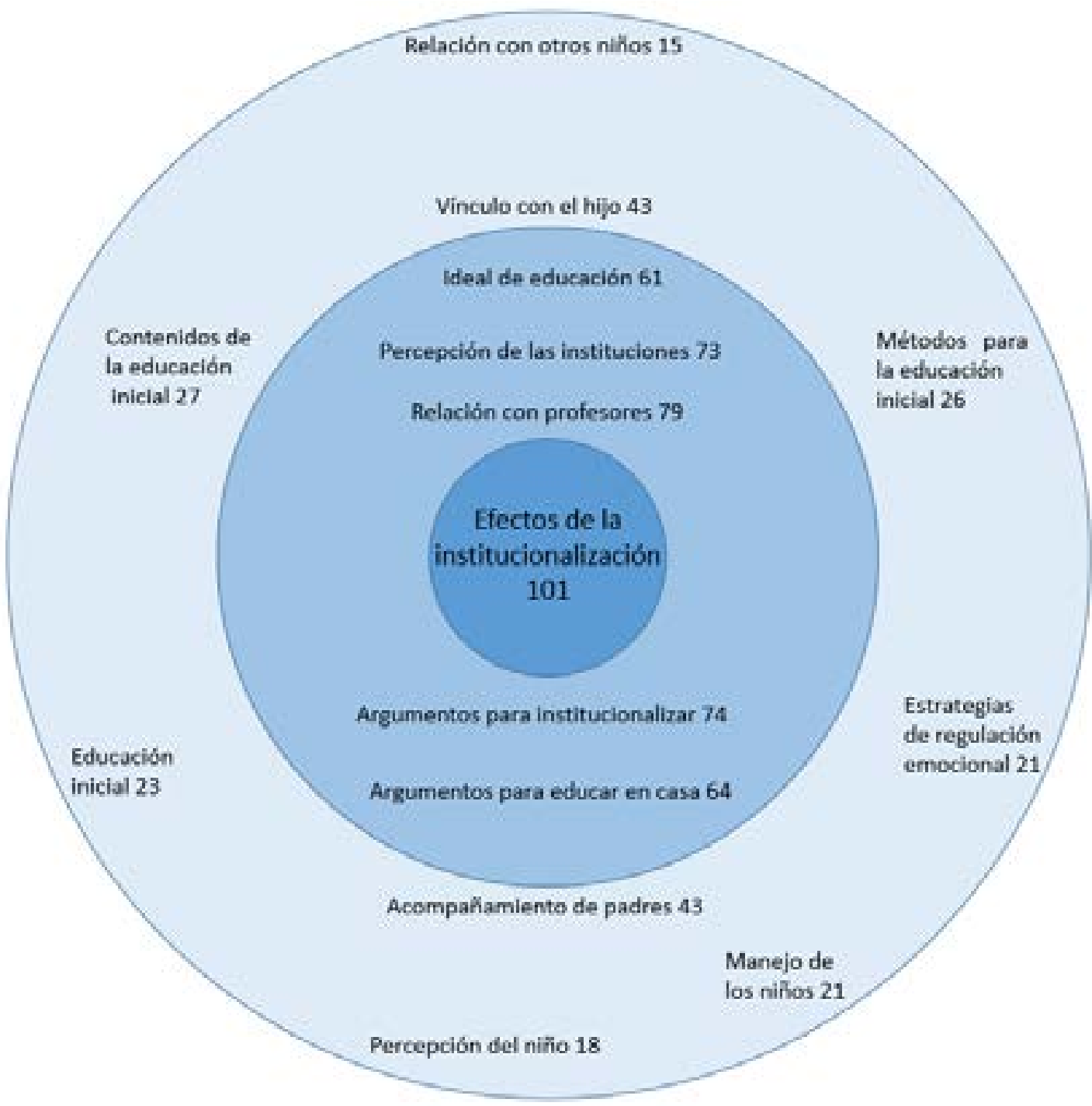

Figura 2. Representaciones sociales de la educación inicial

\section{NÚCLEO CENTRAL}

\section{EFECTOS DE LA INSTITUCIONALIZACIÓN}

Los padres de familia reconocen que después de tener a sus hijos institucionalizados, el lenguaje de los niños es más claro, más fluido y el léxico es más amplio ("Ios niños aprenden a hacerse entender mejor" informante 5, mujer de 24 años). Igualmente, los participantes resaltan que sus hijos han adquirido independencia ("aprendió a comer solito" informante 16, mujer de 23 años) y han asumido responsabilidades en la casa derivadas de las actividades que realizan en la institución ("en el jardín aprenden a ordenar lo que desordenan" informante 3, mujer de 26 años). Los padres de familia hacen énfasis en el buen trato que los niños reciben de parte de las profesoras y en la práctica de los valores que los niños van interiorizando al asistir a las instituciones ("los niños aprenden sus derechos y los de los demás" informante 8, mujer de 25 años), resaltando que observan que los niños lo han interiorizado porque en la casa se reflejan estos aprendizajes en las relaciones intrafamiliares, así como hacen alusión a los conceptos que los niños adquieren ("yo veo muchas habilidades en mi niño que aprendió en el jardín" informante 15, mujer de 23 años), 
aunque refuerzan que lo más importante es que aprendan acerca de sí mismos y de su relación con los demás ("ella aprendió a tratar bien a la gente" informante 12, mujer de 23 años).

Un común denominador en los padres de familia que tienen a sus hijos inscritos en instituciones que ofrecen educación inicial son los aprendizajes que ellos como padres han tenido con relación a la educación de sus hijos ("nos enseñan a leer con los niños" informante 4, mujer de 22 años), así mismo valoran positivamente los encuentros que tienen con otros padres y con profesionales que orientan el trabajo en casa ("nos enseñan a los papás cómo vincularnos con los niños" informante 4).

\section{PRIMERA PERIFERIA}

\section{RELACIÓN CON PROFESORES}

Todos los padres de familia que participaron en esta investigación tienen una percepción positiva del personal que se encarga de la educación inicial en las instituciones que ofrecen atención a la primera infancia en la ciudad de Medellín, demostrando especial agrado por los profesores de sus hijos y atribuyéndoles una función protectora mientras ellos no pueden estar a cargo de sus hijos ("los profesores son los padres en la escuela" informante 9, mujer de 42 años). Igualmente, admiran la profesión docente y reconocen que los profesores cuentan con recursos pedagógicos que ellos como padres no tienen ("uno no es capaz de enseñar lo que las profes les enseñan" informante 5), también les reconocen el esfuerzo por utilizar metodologías variadas para educar a los niños y planear sus actividades pensando en sus particularidades ("las profes utilizan maneras diferentes para enseñar" informante 5).

Generalmente tienen buenas relaciones con las profesoras ("la profesora tiene el talento para llevar a los niños por buen camino" informante 6 , hombre de 31 años) y valoran el trato que dan a sus hijos ("Ios niños dicen que las profes les enseñan a tratar bien" informante 14, mujer de 44 años), así como también agradecen el acompañamiento que las profesoras les realizan en algunas situaciones que ellos como padres no saben manejar ("las profesoras le enseñan a uno a calmarse" informante 13 , mujer de 25 años). Aunque la profesión docente es bien aceptada por todos los participantes, algunos sugieren algunos aspectos que deben tener en cuenta los profesores mientras atienden a los niños ("las profesoras deben resolverles las preguntas a los niños" informante 18, mujer de 27 años) y ("las maestras deben ser muy tolerantes con los niños" informante 17 , mujer de 38 años), entre otras cualidades que ponen la labor docente en un estatus muy alto dentro de la sociedad porque no se limita a una preparación rigurosa desde un punto de vista académico ("que tenga preparación" informante 12), sino que se convierte en una profesión con muchas exigencias sociales.

\section{ARGUMENTOS PARA INSTITUCIONALIZAR}

Los padres de familia llevan a sus hijos a las instituciones que ofrecen la educación inicial para socializar con otras personas diferentes a los miembros de la familia ("Io llevé a la guardería porque el niño era muy solito en la casa" informante 16), reconocen en las instituciones espacios que permiten a los niños desarrollar su lenguaje ("los niños deben aprender a comunicarse en otro lugar" informante 15), creen que se potencian sus habilidades ("aprovechar las capacidades que tienen los niños en esa edad" informante 12), reconocen que los niños adquieren independencia ("los niños deben aprender a defenderse en otro lugar" informante 15), observan que se establecen rutinas de autocuidado y hábitos de estudio ("los niños en el jardín se vuelven juiciosos" informante 3). Las madres de familia encuentran ayuda para desempeñar su rol de madre ("en los jardines encuentro apoyo de las agentes educativas $y$ 
la psicóloga" informante 2, mujer de 32 años), y dan cuenta de una vida más tranquila desde que sus hijos ingresaron a la institución ("para los padres es más fácil la vida cuando están en el jardín" informante 5).

\section{PERCEPCIÓN DE LAS INSTITUCIONES}

Lo primero que los padres de familia manifiestan es agradecimiento a las instituciones por los servicios que les ofrecen tanto a ellos como padres, como a los niños ("agradezco por el apoyo y la oportunidad de que mis niñas estudien" informante 14), valorando mucho el acompañamiento permanente que hacen a las familias con programas variados en la educación de sus hijos ("la guardería hace foros de cómo manejar situaciones con los niños" informante 4). Las instituciones son miradas como espacios apropiados para los niños, amplios y organizados ("las instituciones son reglamentadas y vigiladas" informante 10 , mujer de 39 años), destacando que se han convertidos en lugares seguros para sus hijos y por esta razón los dejan allí sintiéndose tranquilos ("aunque han sido lugares de guerra, las guarderías cuidan a los niños" informante 14). De esta manera, los participantes tienen manifestaciones positivas frente a dichos lugares ("en el jardín se les da mejor educación" informante 3) y aunque algunos padres manifiestan haber tenido un pensamiento negativo en algún momento, luego fueron cambiando su opinión al observar a su hijo feliz ("yo sabía que en la guardería había virus, pero él tenía que enfrentarlo" informante 16).

\section{ARGUMENTOS PARA EDUCAR EN CASA}

Los padres de familia que optaron por la educación en casa nombran actividades que desarrollan dentro del hogar diariamente $y$ que ellos mismos denominan como educativas ("le leemos cuentos diarios en las noches" informante 10), algunas expresiones dan cuenta de temores que tienen los padres con relación a la separación de sus hijos y a dejarlos al cuidado de otras personas ("soy una mamá muy sensible y sobreprotectora" informante 15). Aunque fue común en otras madres escuchar desconfianza para dejar a sus hijos en las instituciones los primeros días, con el paso del tiempo lo fueron superando, pero las madres que le daban mayor relevancia al vínculo con sus hijos argumentaron la importancia de tenerlos en casa durante la primera infancia aprovechando los recursos familiares para ofrecer una educación inicial de calidad ("la niña no tiene tantos juguetes, tiene elementos para crear" informante 10). En esta misma línea, una minoría argumenta la importancia de la educación en casa, a partir de una percepción negativa que tienen con relación a las instituciones que educan a los niños pequeños ("no he tenido buenas experiencias con las guarderías" informante 20 , mujer de 43 años) y por esta razón uno de los padres se encarga de la educación y el cuidado de los niños durante la primera infancia, mientras el otro trabaja para sostener el hogar económicamente.

\section{IDEAL DE EDUCACIÓN}

Los padres de familia tienen dos puntos de vista, uno relacionado con los aspectos positivos que han encontrado en la educación inicial de sus hijos ("se debe enseñar con amor, paciencia y disciplina" informante 1, mujer de 27 años) y otro que se basa en lo que ellos se imaginan de acuerdo a los recuerdos que tienen de su infancia ("la educación inicial debe permitir que la niña sea feliz" informante 11, mujer de 37 años) y ("la educación inicial tiene que estar vinculada con la familia" informante 11). Algunos manifiestan lo que creen que es conveniente para los hijos en la actualidad ("a los niños se les debe poner a utilizar la mente" informante 15).

\section{SEGUNDA PERIFERIA}

\section{ACOMPAÑAMIENTO DE PADRES}

Los padres de familia destacan algunas prácticas 
que realizan ellos con los niños en la casa con la intención de complementar la educación de sus hijos ("he aprendido que yo debo reforzar lo que les enseñan en la institución" informante 7, mujer de 38 años). Estas acciones son reforzadas con redes de apoyo familiar que se vinculan con actividades de cuidado, diversión y direccionamiento de la educación inicial ("los miembros de la familia jugamos mucho con la niña" informante 10). Los padres de familia manifiestan satisfacción por haber dedicado algún tiempo a su hijo ("mi hijo es feliz cuando yo voy a una reunión" informante 7) así como reconocen la importancia que esta acción tiene para los niños y la relacionan con la experiencia que ellos tuvieron en el pasado con sus padres ("mi papá siempre estuvo muy presente en mi infancia" informante 10). Algunos participantes resaltan situaciones que se viven con los niños en los hogares cuando no pueden estar pendientes de su cuidado y, por esta razón, los inscriben en actividades deportivas y culturales cuando no se encuentran escolarizados ("los padres desean ocupar a los niños todo el tiempo" informante 10).

\section{VÍNCULO CON EL HIJO}

La mayoría de los testimonios que conforman esta categoría pertenecen a padres de familia que tienen a sus hijos en instituciones privadas o que optaron por la educación en casa, demostrando un apego fuerte entre padres e hijos ("el vínculo con la mamá es necesario y no debe romperse tan temprano" informante 10), justifican las decisiones que han tomado con respecto a la educación de sus hijos manifestando particularidades de acuerdo a las dinámicas familiares ("el lenguaje de mi niña no requería socialización" informante 10). Resaltan la felicidad, la comodidad y la tranquilidad de sus hijos y manifiestan que sus decisiones se han definido teniendo en cuenta situaciones y espacios que proporcionen el bienestar que la familia necesita ("en este momento estamos tranquilos porque le damos a la niña lo que necesita" informante 10).

Los padres de familia que institucionalizan a sus hijos reconocen que para ellos ha sido muy difícil la separación temprana entre padres e hijos ("yo le muestro debilidad a mi hijo cuando lloro para dejarlo" informante 15), pero con el tiempo encuentran tranquilidad. Se destacan comentarios que realizaron algunos padres de familia evocando su infancia y la relación que tuvieron con sus padres durante los primeros años de vida, recordando momentos felices que compartieron en familia ("cuando mi papá llegaba temprano a casa jugaba con los hijos" informante 10) y a partir de estos testimonios, se motivan a narrar sus experiencias como padres de familia, destacando las transformaciones que han tenido sus vidas desde que se convirtieron en padres ("Ios niños le enseñan a uno más de lo que uno les enseña a ellos" informante 18).

\section{CONTENIDOS DE LA EDUCACIÓN INICIAL}

Los padres de familia se refieren a lo que sus hijos han aprendido desde que están institucionalizados ("les enseñan a ser más seguros de sí mismos" informante 7), ("les explican sobre su cuerpo" informante 13) reconociendo que los logros se deben a las personas que ofrecen el servicio, y también manifiestan lo que ellos consideran que se debe enseñar de acuerdo con la edad de los niños ("enseñarle a los niños a enfrentar diferentes situaciones" informante 8). Clasificaron los contenidos de la educación inicial en dos grandes grupos: el primero tiene que ver con formación en valores ("enseñar el respeto y la solidaridad" informante 18) y el segundo se relaciona con los conceptos que los niños deben manejar en esta etapa ("se le debe enseñar a los niños colores y vocales" informante 15), así como le dan mucha importancia a las habilidades que se deben desarrollar ("enseñar a recortar, a pegar y a hacer círculos" informante 13). 


\section{MÉTODOS PARA LA EDUCACIÓN INICIAL}

Aparecen diferentes técnicas que los padres de familia han utilizado en casa para educar a sus hijos ("dejar que los niños experimenten, toquen y miren" informante 8), así como también valoran positivamente las estrategias que utilizan en las instituciones que ofrecen educación inicial ("he aprendido que con los cuentos los niños aprenden" informante 4) y ("a los niños se les debe enseñar con juegos" informante 17). Al desarrollar el grupo focal, se genera una discusión con relación a los métodos que se utilizaban anteriormente, donde los niños en la primera infancia eran muy pasivos e interactuaban poco con los adultos ("ahora se enseña diferente a lo que me tocó a mí" informante 7) mientras que en la actualidad, los niños tienen más oportunidades tanto en las instituciones como en la casa ("se trabaja a partir de los intereses de los niños" informante 8). Aunque se noten diferencias en los tiempos, los participantes rescatan que los métodos que se utilizan con los niños en cualquier época dejan experiencias muy significativas para la vida ("tengo recuerdos muy bonitos con muñequitos, títeres y plastilina" informante 5) y ("uno le va viendo evolución a los niños con las metodologías de las profes" informante 4).

\section{EDUCACIÓN INICIAL}

Definida por los padres de familia como básica dentro del proceso de formación de todo niño ("es la base para desarrollar sus capacidades" informante 12) y creen que marca la ruta a seguir en los próximos años y que es importante en la vida de sus hijos ("es un momento donde el niño capta todo lo del ambiente" informante 12). La percepción acerca de la educación inicial es positiva y valoran los efectos que ésta tiene en sus hijos ("sirve para que los niños se vuelvan curiosos" informante 1) y ("esta educación es para reforzar la inteligencia" informante 20). Los participantes perciben la educación inicial en los mismos términos que ha sido comprendida la educación preescolar en Colombia durante varios años, asumiéndola como preparación para etapas posteriores ("esta educación les va a servir a las niñas para más adelante" informante 14), dando a entender que lo que viven los niños en su educación inicial cobrará mayor relevancia en un futuro ("la educación inicial prepara para lo que sigue" informante 13), así mismo, la ubican fuera de casa y la ponen en manos de otras personas como profesores y psicólogos.

\section{ESTRATEGIAS DE REGULACIÓN EMOCIONAL}

A través de la educación inicial, los padres de familia adquieren herramientas para manejar situaciones que viven en casa con sus hijos, por ejemplo cuando se sienten inseguros para realizar las actividades ("darles confianza a los niños" informante 4), cuando los hijos pierden el interés por las actividades que hacen ("a manejar la frustración" informante 8) y cuando los padres deben explicar varias veces las mismas instrucciones ("aprendemos a tener más paciencia con los niños" informante 5). Según los padres de familia, los mismos niños aprenden a manejar sus emociones desde que reciben educación inicial ("a los niños les enseñan mucho los valores" informante 15) y cuando llegan a casa, les enseñan a sus hermanos y a sus padres todo lo que aprenden diariamente en la institución.

\section{MANEJO DE LOS NIÑOS}

Los participantes describen el trato que se les debe dar a los niños a partir de las vivencias que han tenido con sus hijos en los primeros años de vida ("a los niños hay que explicarles todo" informante 7), sugieren algunas prácticas que ellos consideran que pueden ayudar a los niños ("a los niños hay que tenerles mucha paciencia" informante 19, mujer de 45 años) y ("a los niños se les debe ir enseñando sin forzarlos mucho" informante 20). Cabe anotar que los padres que 
se atrevieron a dar sugerencias con respecto a la educación de los niños lo hicieron al final de la entrevista, cuando ya estaban en un estado de mayor confianza y tranquilidad. Así mismo, algunas madres que apenas inician el proceso de escolarización con sus hijos terminaron la entrevista angustiadas porque se sienten muy solas en la educación de sus hijos ("no sé cómo manejar situaciones con mis hijas" informante 8) y expresan que, aunque cuenten con una pareja, la tarea de la educación de sus hijos les corresponde a ellas solas.

\section{PERCEPCIÓN DEL NIÑO}

Algunos padres de familia expresan que su percepción del niño ha ido cambiando desde que sus hijos reciben educación inicial ("el niño es cada día más maduro" informante 16) y se entrevé una nueva valoración al niño que se relaciona con la contemporaneidad y con los modelos educativos que se encuentran en la ciudad ("en este momento los niños necesitan más juego" informante 19) y ("ahora les podemos exigir más a los niños académicamente" informante 7). En síntesis, el concepto de niño que tienen los padres de familia es: un sujeto curioso, inteligente, responsable, con capacidad para expresarse y para relacionarse con otras personas, necesitado de juego y en ocasiones con comportamientos difíciles.

\section{RELACIÓN CON OTROS NIÑOS}

Lospadres defamiliaaprecianmucholosespacios de socialización entre niños porque descubren que sus hijos aprenden a relacionarse con otras personas mientras practican diferentes valores ("los niños aprenden a convivir en la guardería" informante 5), así como asimilan nuevos conceptos ("los niños aprenden a intercambiar conocimientos con otros niños" informante 6). Destacan la influencia que ejercen otros niños sobre sus hijos y que se refleja en el cambio de actitud frente a las actividades escolares ("los niños se entusiasman aprendiendo con otros niños" informante 16) y en la manera como sus hijos van aprendiendo a reaccionar frente a diferentes situaciones que se les presentan tanto en la casa, como en contextos sociales ("la educación inicial le sirve para aprender a manejar situaciones con otros niños” informante 8).

\section{DISCUSIÓN}

Las RS de este estudio tienen como elemento periférico los contenidos de la educación inicial y lo central son los efectos que tiene la institucionalización, mientras que en otros estudios lo principal son los contenidos que se deben enseñar (Fontaine y Hamon, 2010; Limón-Aguirre, 2011; Räty y Snellman, 1998; Räty, Komoulainen y Hirva, 2012; RogeroGarcía y Candelas, 2015). Pareciera que las RS nuestras están marcadas por un enfoque más funcionalista de la educación inicial mientras que en los antecedentes, dichas RS están marcadas por una perspectiva estructuralista centrada en los contenidos de la educación inicial y no en sus fines.

Sin embargo, estos efectos de la institucionalización (núcleo central) están ligados a los aprendizajes que adquieren los niños con la educación, que van más allá de contenidos académicos, que, si bien son importantes, los padres de familia le asignan mayor valor a la autonomía, a la independencia, a la práctica de valores, al desarrollo del lenguaje y a la socialización que logran sus hijos cuando asisten a una institución que ofrece educación inicial.

Para los padres de familia el desarrollo de competencias y el éxito académico y profesional que tendrán sus hijos en el futuro se relaciona con la educación inicial que reciben los niños en instituciones externas a la casa; demostrando que sus RS con respecto a la educación inicial están determinadas por los conocimientos y las experiencias que han tenido de las instituciones que atienden a la primera infancia y por la 
situación de empleabilidad que viven los padres actualmente ${ }^{5}$.

Teniendo en cuenta que las familias pertenecen a una sociedad laboral, las RS de la educación inicial están influenciadas por el trabajo que desempeñan tanto padres como madres de familia, pues sus exigencias laborales y de consumo, limitan el tiempo que comparten con sus hijos haciendo que perciban a la educación inicial como aquella que se ofrece en las instituciones que atienden a los niños, delegando la tarea de educar a los profesores.

A partir de los hallazgos de esta investigación, surge una hipótesis con relación a la estructura de las RS, definiéndolas como constructos que las personas hacen a partir de la información que han recibido de diferentes fuentes y que a través de un proceso de abstracción de un fenómeno, elaboran mentalmente un concepto que es definido teniendo en cuenta la experiencia que se vive, es decir, que para el caso de los padres de familia las RS acerca de la educación inicial están constituidas por la información relacionada con el tema que el medio ha puesto en ellos y que es complementada con la experiencia educativa que viven actualmente con sus hijos.

El origen de toda representación parece estar ligado a las prácticas sociales, Flament y Rouquette (2003) establecieron cuatro prácticas ligadas a la representación social: el desempeño de la acción, la intensidad de la ejecución, la experticia en la acción y las diferentes formas de ejecutar una acción. En este caso, podría pensarse que las prácticas sociales de la educación inicial han ido aumentando y generalizándose en la población, convirtiéndose en una necesidad para todos los niños desde los primeros años de vida, que es comprendida por los adultos como una obligación y es atendida y vigilada por el estado. Así mismo, se han ido ampliando las áreas de atención y las personas

$5 \quad$ https://www.dane.gov.co/index.php/estadisticaspor-tema/mercado-laboral/empleo-y-desempleo que están a cargo van profesionalizando continuamente su acción, ofreciendo variadas estrategias de acuerdo a las características y demandas de los niños.

Otra posible explicación de la configuración de la RS de la educación inicial se daría a partir del modelo de los Esquemas Cognitivos de Base (schèmes cognitifs de base - SCB). Rateau (1995) estableció que habrían tres metaesquemas cognitivos de base que conformarían la RS: descripción, praxis y evaluación, que luego fueron reformulados por Flament (1994) y Abric y Tafani (1995) abordando un modelo de dos dimensiones: una normativa y otra práctica. De acuerdo a los testimonios de los padres de familia entrevistados, sus RS acerca de la educación inicial tienen un componente normativo que ha sido impuesto por el estado y que hace que esta educación sea un derecho adquirido para los niños donde ellos como padres deben buscar los medios para garantizarlo, mientras que la sociedad ha influido en la estructuración de la RS, mostrando la educación inicial como una actividad lúdica que le permite a los niños explorar, aprender y ser felices en espacios seguros y con las condiciones necesarias para su bienestar.

\section{CONCLUSIONES}

Las RS de los padres de familia acerca de la educación inicial en la ciudad de Medellín son positivas y las instituciones que atienden a los niños en su primera infancia se han convertido en un espacio seguro para ellos, que en algunos casos puede ofrecer más garantías de derechos que las que tienen en sus propios hogares.

Sin embargo, es importante poner en tensión esta representación de los padres de familia ya que la calidad de los jardines de cuidado infantil de numerosos países de Latinoamérica, ha sido medida por la observación directa de los centros, encontrándose que la calidad con respecto a las interacciones entre cuidadores y niños sigue 
siendo baja. Berlinski y Schady (2015) exponen lo que ocurre en países que proporcionan dicho servicio básicamente a través de la modalidad comunitaria (como Colombia y Perú), aquellos que utilizan la modalidad institucional (como Brasil y Chile) y aquellos que ofrecen una combinación de ambas modalidades (como Ecuador), anotando que han sido evaluados con diferentes pruebas que miden la calidad del servicio y siempre han puntuado muy bajo ( $p$. 112).

En Colombia se han realizado estudios que evalúan las reformas efectuadas a los hogares comunitarios tanto a nivel de infraestructura, como evaluando la iniciativa de capacitar y otorgar algún certificado en desarrollo infantil para las madres de la comunidad que trabajaban como cuidadoras de los niños, formándolas en salud infantil, nutrición y desarrollo, hitos del desarrollo, prácticas educativas y estimulación para niños; sin embargo, la calidad del proceso con los niños no mejoró, no se observaron cambios en la nutrición de los niños, así como tampoco hubo avances en su desarrollo cognitivo ni socioemocional (Berlinski y Schady, 2015, p. 115).

Queda entonces un gran interrogante con relación a lo que significa calidad educativa para los padres de familia, porque evidentemente sus RS se encuentran muy alejadas de las investigaciones de múltiples evaluaciones sobre el impacto de los servicios de los jardines de cuidado infantil en el desarrollo del niño en América Latina y el Caribe, que muestran limitaciones metodológicas y bajos estándares de calidad en los niveles de cumplimiento y peor aún, los jardines de cuidado infantil de jornada completa no mejoran de manera consistente el desarrollo infantil (Berlinski y Schady, 2015, p. 116).

Teniendo en cuenta que las RS que los padres de familia tienen con relación a los profesores que atienden a sus hijos son favorables, se debe fortalecer la formación de los agentes educativos y profesores de primera infancia, teniendo en cuenta las características de los niños de la ciudad y de sus familias, pues se encontró que aunque pertenezcan a diferentes zonas, las familias demandan profesores que conozcan muy bien el comportamiento de los niños en la primera infancia, que puedan brindar educación en valores y que ofrezcan elementos pedagógicos que ellos como padres no poseen. En consecuencia, la secretaría de educación de la ciudad debe continuar garantizando la inversión y la atención por parte del estado en el trabajo con la primera infancia y, de ser posible, ampliar su cobertura en todas las zonas en las que se divide el territorio.

Los padres de familia valoran mucho la capacitación que reciben de las instituciones de educación inicial y consideran que esta formación contribuye en sus vidas para ser mejores padres y educar a los niños en coherencia con la política de educación inicial, por lo tanto, se requiere ampliar el programa de acompañamiento a los padres para tener mayor impacto en las familias y en la educación que ofrecen a los niños mientras se encuentran en casa.

Al realizar el trabajo de campo, se tuvo contacto con padres de familia de diferentes zonas de la ciudad de Medellín sin encontrar diferencias significativas de acuerdo con el lugar en sus RS acerca de la educación inicial de los niños, esto podría deberse a la alta movilidad interna entre los barrios de la ciudad. Sin embargo, fue una muestra baja con relación al número de habitantes de la ciudad, por lo que se propone para futuras investigaciones incluir una muestra mayor.

Con el fin de ampliar la categoría del núcleo central (efectos de la institucionalización) se propone realizar una investigación longitudinal con los niños que han estado inmersos desde temprana edad en la escolarización 
formal, de esta manera se podría contrastar si esta representación de los efectos de la institucionalización se mantiene a lo largo de la primera parte del ciclo vital, esto es, hasta la culminación de los estudios básicos.

Finalmente, se debe auditar la conservación de los contenidos puesto que existe una buena valoración de sus efectos, esto sería posible con el fortalecimiento de los observatorios en primera infancia conformados por profesores, padres de familia, instituciones que ofrecen programas de formación docente para la primera infancia y expertos en educación inicial.

\section{FINANCIACIÓN}

Esta investigación fue realizada en el marco del doctorado en Ciencias de la Educación de la Universidad de San Buenaventura, sede Medellín y fue financiada por recursos propios de la investigadora principal, así como por la Secretaría de Educación de Medellín y la Universidad de San Buenaventura.

\section{Agradecimientos}

Se agradece especialmente a SAPIENCIA, entidad dependiente de la Secretaría de Educación de Medellín, por la asignación de una beca parcial a la autora principal, esto facilitó enormemente la consolidación del trabajo. De igual manera, se agradece a los padres de familia que participaron voluntariamente en el trabajo de campo.

\section{REFERENCIAS BIBLIOGRÁFICAS}

Abric, J. (2001). Prácticas sociales y representaciones. México: Ediciones Coyoacán.

Abric, J. y Tafani, E. (1995). Nature et fonctionnement du noyau central d'une représentation sociale: la représentation de l'entreprise. Cahiers Internationaux de Psychologie Sociale, 28, 22-31.

Berlinski, S. y Schady, N. (2015). Los primeros años: el bienestar infantil y el papel de las políticas públicas. Washington, D.C.Banco Interamericano de Desarrollo

Cerda, H. (1996). Educación preescolar: Historia, legislación, currículo y realidad económica. Colombia: Cooperativa Editorial Magisterio.

Congreso de la república de Colombia. Ley 1090 de 2006. Recuperado de http:// www.secretariasenado.gov.co/senado/ basedoc/ley 1090 2006.html

Durkheim, E. (2001) segunda reimpresión de la primera edición en español. Las reglas del método sociológico. México: Fondo de cultura económica.

Flament, C. (1994). Structure, dynamique et transformation des representations sociales. In J. C. Abric (Ed.), Pratiques sociales et representations. Paris: PUF.

Flament, C. y Rouquette, M. L. (2003). Anatomie desidées ordinaires. Paris: Armand Colin.

Fontaine, S. yHamon, J. (2010). La représentation sociale de l'école des parents et des enseignants à La Réunion. Les Cahiers Internationaux de Psychologie Sociale, 85, 69-109. DOI 10.3917/cips.085.0069 
Limón-Aguirre, F. (2011). Representaciones sociales de la educación escolar entre los Chuj Mexicanos. Revista Pueblos y Fronteras Digital, 6(12), 133-166.

MEN, Decreto número 088 de 1976, Recuperado de https://www.mineducacion.gov. co/1759/articles-102584_archivo_pdf.pdf

MINSALUD, Resolución número 8430 de 1993. Recuperado de https://www.minsalud.gov. $\mathrm{co/sites/rid/Lists/BibliotecaDigital/RIDE/}$ DE/DIJ/RESOLUCION-8430-DE-1993. pdf

Morgan, D. (1997). Focus Group as Qualitative Research. 2ed. Londres: Sage.

Morgan, D. y Krueger, R. (1998). The Focus Group Kit. Londres: Sage.

Moscovici, S. (1979). El psicoanálisis, su imagen y su público. Argentina: Huemul.

Rateau, P. (1995). Le noyau central des représentations sociales comme système hiérarchisé. Une étude sur la représentation du groupe. Cahiers Internationaux de Psychologie Sociale, 26, 29-52.

Rateau, P., Moliner, P., Guimelli, C., Abric, J.-C. (2011). Social Representation Theory. En P. Van Lange, A. Kruglanski, T. Higgins (Eds.), Handbook of Theories of Social Psychology vol.2 (pp. 478-498). London, Thousand Oaks, CA: Sage.

Räty, H. y Snellman, L. (1998). Social Representations of Educability. Social Psychology of Education, 1(4), 359-373. Doi: 10.1007/BF02335554

Räty, H., Komulainen, K. y Hirva, L. (2012). Social representations of educability in Finland: 20 years of continuity and change. Social Psychology of Education, 15(3), 395-409. DOI 10.1007/s11218-012-9188-0
Restrepo-Ochoa, D.A. (2013). La Teoría Fundamentada como metodología para la integración del análisis procesual y estructural en la investigación de las Representaciones Sociales. Revista CES Psicología, 6(1), 122-133.

Rogero García, J. y Candelas, M. (2015). Representaciones sociales de los padres y madres sobre la educación pública y privada en España. Revista de la Asociación de Sociología de la Educación, 9(1), 46-58.

Wachelke, J. (2012). Social Representations: A Review of Theory and Research from the Structural Approach. Universitas Psychologica, 11(3), 729-741. 